\title{
COMPLEX ANALYTIC CONNECTIONS IN FIBRE BUNDLES $\left({ }^{1}\right)$
}

\author{
BY \\ M. F. ATIYAH
}

Introduction. In the theory of differentiable fibre bundles, with a Lie group as structure group, the notion of a connection plays an important role. In this paper we shall consider complex analytic connections in complex analytic fibre bundles. The situation is then radically different from that in the differentiable case. In the differentiable case connections always exist, but may not be integrable; in the complex analytic case connections may not exist at all. In both cases we are led therefore to certain obstructions, an obstruction to the integrability of a connection in the differentiable case, an obstruction to the existence of a connection in the complex analytic case. It is a basic theorem that, if the structure group is compact, the obstruction in the differentiable case (the curvature) generates the characteristic cohomology ring of the bundle (with real coefficients). What we shall show is that, in a large class of important cases, the obstruction in the complex analytic case also generates the characteristic cohomology ring. Using this fact we can then give a purely cohomological definition of the characteristic ring. This has a number of advantages over the differentiable approach: in the first place the definition is a canonical one, not depending on an arbitrary choice of connection; secondly we remain throughout in the complex analytic domain, our characteristic classes being expressed as elements of cohomology groups with coefficients in certain analytic sheaves; finally the procedure can be carried through without change for algebraic fibre bundles.

The ideas outlined above are developed in considerable detail, and they are applied in particular to a problem first studied by Weil [17], namely the problem of characterizing those fibre bundles which arise from a representation of the fundamental group. We show how Weil's main result fits into the general picture, and we discuss various aspects of the problem.

As no complete exposition of the theory of complex analytic fibre bundles has as yet been published, this paper should start with a basic exposition of this nature. However this would be a major undertaking in itself, and instead we shall simply summarize in $\$ 1$ the terminology and results on vector bundles which we require, and for the rest we refer to Grothendieck [8], Serre [12], and Hirzebruch [9].

Received by the editors June 21, 1956.

(1) This work was done while the author was a Commonwealth Fund Fellow. 


\section{Contents}

Introduction

1. Vector bundles and sheaves

2. Complex analytic connections

3. The characteristic ring

4. The $D$-functor

5. Chern classes

6. Line-bundles

7. Representations of the fundamental group

8. Higher dimensional varieties

1. Vector bundles and sheaves. Throughout this paper we shall be concerned with complex analytic structures, and where no ambiguity can arise we shall omit the words "complex analytic."

Let $X$ be a complex analytic manifold. By a vector bundle over $X$ we shall always mean a complex analytic vector bundle, that is the fibre is a complex vector space and the structure group is the general linear group (cf. [13]). Let $O$ be the sheaf of germs of holomorphic functions on $X$, and let $E$ be a vector bundle over $X$. Then the sheaf of germs of (complex analytic) cross-sections of $E$ is a coherent sheaf of $O$-modules (in the sense of Serre [12]), and we denote it by $E$. Since $E$ is locally trivial it follows that $E$ is locally free; more precisely, for each $x \in X$, there exists an open neighborhood $U$ such that

$$
E\left|U \cong O^{r}\right| U,
$$

where $O^{r}$ is the direct sum of $r$ copies of $O$, and the isomorphism is an $O$ isomorphism. Conversely, if $S$ is a locally free sheaf of $O$-modules on $X$, and if $X$ is connected, there exists a vector bundle $E$ on $X$ such that $S \cong E$ (cf. $[12]) . E$ is determined up to an isomorphism by $S$. We shall denote by $\Gamma(E)$ the vector space of cross-sections of $E$. Then we have

$$
\Gamma(E)=H^{0}(X, E) .
$$

If $E, F$ are vector bundles over $X$ we can define the vector bundles $E^{*}$, $E \oplus F, E \otimes F$, Hom $(E, F)$, whose fibres at $x$ are the vector spaces $E_{x}^{*}, E_{x} \oplus F_{x}$, $E_{x} \otimes_{C} F_{x}, \operatorname{Hom}_{C}\left(E_{x}, F_{x}\right)$ (cf. [9]). Then we have the canonical isomorphism $\operatorname{Hom}(E, F) \cong E^{*} \otimes F$. In particular, if 1 denotes the trivial line-bundle $\left({ }^{2}\right)$, $\operatorname{Hom}(E, 1) \cong E^{*}$.

If $R, S$ are coherent sheaves on $X$ we can define the sheaves $R \oplus S, R \otimes S$, Hom $(\boldsymbol{R}, \boldsymbol{S})$ whose stalks at $x$ are the $O_{x}$-modules $\boldsymbol{R}_{x} \oplus \boldsymbol{S}_{x}, \boldsymbol{R}_{x} \otimes_{O_{x}} \boldsymbol{S}_{x}$, $\operatorname{Hom}_{\boldsymbol{O}_{x}}\left(\boldsymbol{R}_{x}, \boldsymbol{S}_{x}\right)$ (Serre [12]). In particular the operations $\oplus, \otimes$, Hom can be applied to the locally free sheaves $E, F$. It is then easily verified that $E \oplus F, E \otimes F$, Hom $(E, F)$ are canonically isomorphic with the sheaves of

(2) A line-bundle is a vector bundle in which the fibre is a one-dimensional vector space. 
germs of cross-sections of $E \oplus F, E \otimes F$, Hom $(E, F)$, so that our notation is consistent. In particular if $F=1$, then $F=O$, and $\operatorname{Hom}(E, O)$ is the sheaf of germs of cross-sections of $\operatorname{Hom}(E, 1)$. Hence, if we denote this sheaf by $E^{*}$, we can identify $E^{*}$ with the sheaf of germs of cross-sections of $E^{*}$.

Besides the operations just defined we can also define the vector bundle $\wedge^{p} E$ whose fibre at $x$ is the vector space $\wedge^{p} E_{x}$ (cf. [9]). If in particular we take $p=r$, where $E_{x}$ is $r$-dimensional, we shall write $\operatorname{det} E$ instead of $\AA^{r} E$. $\operatorname{det} E$ is thus a line-bundle, and it is just the line-bundle induced from $E$ by the homomorphism det: $G L_{r} \rightarrow C^{*}$. We denote by det $E$ the sheaf of germs of crosssections of $\operatorname{det} E$.

By a homomorphism $h: E \rightarrow F$ we shall mean an element $h \in \Gamma$ Hom $(E, F)$; $h$ is thus a complex analytic mapping of $E$ into $F$, such that $h_{x}: E_{x} \rightarrow F_{x}$ is a homomorphism of vector spaces for each $x$. If $F=E$ we write End $E$ instead of Hom $(E, E)$, and $\Gamma$ End $E$ is then the vector space of endomorphisms of $E$. From the canonical isomorphism

$$
\Gamma \operatorname{Hom}(E, F) \cong H^{0}(X, \operatorname{Hom}(E, F)),
$$

it follows that $h: E \rightarrow F$ corresponds to a sheaf homomorphism $h: E \rightarrow F$.

Definition. $0 \rightarrow E^{\prime} \rightarrow^{h} E \rightarrow^{0} E^{\prime \prime} \rightarrow 0$ is an exact sequence of vector bundles if $0 \rightarrow E_{x}^{\prime} \rightarrow^{h} E_{x} \rightarrow \boldsymbol{o}_{x} E_{x}^{\prime \prime} \rightarrow 0$ is an exact sequence of vector spaces for each $x \in X . E^{\prime}$ is called a sub-bundle of $E$, and $E^{\prime \prime}$ a factor bundle of $E$.

Definition. An exact sequence (of vector bundles, sheaves,...) $0 \rightarrow A \rightarrow^{\prime} B \rightarrow^{\circ} C \rightarrow 0$ is split if there exists a homomorphism $h: C \rightarrow B$ such that $g h=1: C \rightarrow C$. The homomorphism $h$ is then said to be a splitting of the exact sequence.

If $0 \rightarrow A \rightarrow B \rightarrow C \rightarrow 0$ is split, then $B$ is a direct sum; more precisely $B=f A \oplus h C$.

Proposition 1. $0 \rightarrow E^{\prime} \rightarrow E \rightarrow E^{\prime \prime} \rightarrow 0$ is an exact sequence of vector bundles if and only if $0 \rightarrow E^{\prime} \rightarrow E \rightarrow E^{\prime \prime} \rightarrow 0$ is an exact sequence of sheaves.

Proof. If the vector bundle sequence is exact we obtain the exact sequence of sheaves immediately. We have to prove the converse. Suppose therefore that $0 \rightarrow E^{\prime} \rightarrow E \rightarrow E^{\prime \prime} \rightarrow 0$ is exact. Since $E^{\prime \prime}$ is locally free we may lift the local generators of $E^{\prime \prime}$ in the neighborhood of any point $x$ back into $E$ to give a local splitting of the exact sequence. That is, every $x$ has an open neighborhood $U$ such that $E_{U}=f E_{U}^{\prime} \oplus \boldsymbol{h} E_{U}^{\prime \prime}$, where $E_{U}$ denotes the restriction of $E$ to $U$ and $h: E_{U}^{\prime \prime} \rightarrow E_{U}$ is a local splitting. Then $E_{U}=f E_{U}^{\prime} \oplus h E_{U}^{\prime \prime}$, and so $E_{x}$ $=f_{x} E_{x}^{\prime} \oplus h_{x} E_{x}^{\prime \prime}$. Hence $0 \rightarrow E_{x}^{\prime} \rightarrow E_{x} \rightarrow E_{x}^{\prime \prime} \rightarrow 0$ is exact for all $x$, and therefore $0 \rightarrow E^{\prime} \rightarrow E \rightarrow E^{\prime \prime} \rightarrow 0$ is exact.

If $0 \rightarrow E^{\prime} \rightarrow E \rightarrow E^{\prime \prime} \rightarrow 0$ is an exact sequence of vector bundles, then we have proved that $E$ is locally isomorphic with $E^{\prime} \oplus E^{\prime \prime}$. Moreover, if

$$
\rho: E_{U}^{\prime} \oplus E_{U}^{\prime \prime} \rightarrow E_{U}, \quad \sigma: E_{U}^{\prime} \oplus E_{U}^{\prime \prime} \rightarrow E_{U}
$$


are two local isomorphisms, the difference

$$
\sigma^{-1} \rho: E_{U}^{\prime} \oplus E_{U}^{\prime \prime} \rightarrow E_{U}^{\prime} \oplus E_{U}^{\prime \prime}
$$

is of the form $I+\phi$, where $\phi: E_{U}^{\prime \prime} \rightarrow E_{U}^{\prime}$, and $I$ is the identity. Passing to the sheaves a precisely similar result holds. In the terminology of Grothendieck [8] $E$ is a fibre space of type $E^{\prime} \oplus E^{\prime \prime}$ with structure sheaf Hom $\left(E^{\prime \prime}, E^{\prime}\right)$; here $\operatorname{Hom}\left(E^{\prime \prime}, E^{\prime}\right)$ is identified with a subsheaf of End $\left(E^{\prime} \oplus E^{\prime \prime}\right)$ by $\phi \rightarrow I+\phi$.

Definition. An extension of $E^{\prime \prime}$ by $E^{\prime}$ is an exact sequence (of vector bundles)

$$
0 \rightarrow E^{\prime} \rightarrow E \rightarrow E^{\prime \prime} \rightarrow 0 .
$$

Definition. Two extensions of $E^{\prime \prime}$ by $E^{\prime}$ are equivalent if we have an isomorphism of exact sequences:

$$
\begin{aligned}
0 \rightarrow & E^{\prime} \rightarrow E_{1} \rightarrow E^{\prime \prime} \rightarrow 0 \\
& \downarrow I^{\prime} \quad \downarrow \quad \downarrow \quad \downarrow I^{\prime \prime} \\
0 \rightarrow & E^{\prime} \rightarrow E_{2} \rightarrow E^{\prime \prime} \rightarrow 0
\end{aligned}
$$

where $I^{\prime}, I^{\prime \prime}$ are the identity endomorphisms of $E^{\prime}$ and $E^{\prime \prime}$.

If the exact sequence $0 \rightarrow E^{\prime} \rightarrow E \rightarrow E^{\prime \prime} \rightarrow 0$ splits we say that the extension is trivial. Alternatively a trivial extension is one which is equivalent to a direct sum: $0 \rightarrow E^{\prime} \rightarrow E^{\prime} \oplus E^{\prime \prime} \rightarrow E^{\prime \prime} \rightarrow 0$.

Similar definitions hold for the corresponding sheaves, and by Proposition 1 the extensions of $E^{\prime \prime}$ by $E^{\prime}$ correspond one-to-one to the extensions of $E^{\prime \prime}$ by $E^{\prime}$, equivalence being preserved in this correspondence. In the terminology of [8] equivalent extensions of $E^{\prime \prime}$ by $E^{\prime}$ correspond to isomorphic fibre spaces of type $E^{\prime} \oplus E^{\prime \prime}$, with structure sheaf Hom $\left(E^{\prime \prime}, E^{\prime}\right)$. But the isomorphism classes of such fibre spaces are in one-to-one correspondence with the elements of $H^{1}\left(X\right.$, Hom $\left(E^{\prime \prime}, E^{\prime}\right)$ ) (cf. [8]). Hence we obtain:

Proposition 2. The equivalence classes of extensions of $E^{\prime \prime}$ by $E^{\prime}$ are in one-to-one correspondence with the elements of $H^{1}\left(X, \operatorname{Hom}\left(E^{\prime \prime}, E^{\prime}\right)\right)$, the trivial extension corresponding to the zero element.

REMARK. A corresponding result holds in the differential case, but Hom $\left(E^{\prime \prime}, E^{\prime}\right)$ is then a fine sheaf and so $H^{1}\left(X\right.$, Hom $\left.\left(E^{\prime \prime}, E^{\prime}\right)\right)=0$. Thus every extension is trivial. The same is true if $X$ is a Stein manifold, since Hom $\left(E^{\prime \prime}, E^{\prime}\right)$ is then a coherent sheaf. We are primarily concerned however with the compact complex case, and $H^{1}\left(X\right.$, Hom $\left.\left(E^{\prime \prime}, E^{\prime}\right)\right)$ is then nonzero in general.

For applications we shall require to know the precise element of $H^{1}\left(X\right.$, Hom $\left.\left(E^{\prime \prime}, E^{\prime}\right)\right)$ corresponding to a given extension. This is defined as follows. Operate on the exact sequence $0 \rightarrow E^{\prime} \rightarrow E \rightarrow E^{\prime \prime} \rightarrow 0$ by the functor Hom $\left(E^{\prime \prime}, \quad\right)$; since $E^{\prime \prime}$ is locally free we get an exact sequence 


$$
0 \rightarrow \operatorname{Hom}\left(E^{\prime \prime}, E^{\prime}\right) \rightarrow \operatorname{Hom}\left(E^{\prime \prime}, E\right) \rightarrow \operatorname{Hom}\left(E^{\prime \prime}, E^{\prime \prime}\right) \rightarrow 0 .
$$

The cohomology sequence of this is

$\rightarrow H^{0}\left(X, \operatorname{Hom}\left(E^{\prime \prime}, E\right)\right) \rightarrow H^{0}\left(X, \operatorname{Hom}\left(E^{\prime \prime}, E^{\prime \prime}\right)\right) \stackrel{8}{\rightarrow} H^{1}\left(X, \operatorname{Hom}\left(E^{\prime \prime}, E^{\prime}\right)\right) \rightarrow$.

Then $\delta\left(I^{\prime \prime}\right) \in H^{1}\left(X, \operatorname{Hom}\left(E^{\prime \prime}, E^{\prime}\right)\right)$ is the element given Proposition 2, where as before

$$
I^{\prime \prime} \in \Gamma E \text { End } E^{\prime \prime}=H^{0}\left(X, \text { Hom }\left(E^{\prime \prime}, E^{\prime \prime}\right)\right)
$$

is the identity endomorphism of $E^{\prime \prime}$. We make this more explicit. For a suitable covering $\left\{U_{i}\right\}$ of $X$ the restriction of the given extension to each $U_{i}$ will be trivial; let $h_{i}: E_{i}^{\prime \prime} \rightarrow E_{i}$ be the splitting homomorphisms, where $E_{i}{ }^{\prime \prime}, E_{i}$ denote the restrictions of $E^{\prime \prime}, E$ to $U_{i}$. Then $\left\{\boldsymbol{h}_{i}\right\}$ is a 0 -cochain of Hom $\left(\boldsymbol{E}^{\prime \prime}, \boldsymbol{E}\right)$ which lifts the 0 -cocycle $I^{\prime \prime}$ of Hom $\left(E^{\prime \prime}, E^{\prime \prime}\right)$. Hence $\left\{\boldsymbol{h}_{j}-\boldsymbol{h}_{\boldsymbol{i}}\right\}$ is a representative 1-cocycle for $\delta\left(I^{\prime \prime}\right)$. On the other hand we have the local isomorphisms:

$$
u_{i}: E_{i}^{\prime} \oplus E_{i}^{\prime \prime} \rightarrow E_{i}
$$

defined by $u_{i}\left(\mathbf{s}^{\prime} \oplus \mathbf{s}^{\prime \prime}\right)=\left(\mathbf{s}^{\prime}+h_{i}\left(\mathbf{s}^{\prime \prime}\right)\right)$. Thus $E$ is defined, as a fibre space of type $E^{\prime} \oplus E^{\prime \prime}$ by the coordinate transformations:

$$
\boldsymbol{g}_{i j}=u_{i}^{-1} u_{j}: E_{i j}^{\prime} \oplus E_{i j}^{\prime \prime} \rightarrow E_{i j}^{\prime} \oplus E_{i j}^{\prime \prime},
$$

where $E_{i j}^{\prime}$, $E_{i j}^{\prime \prime}$ denote the restrictions of $E^{\prime}, E^{\prime \prime}$ to $U_{i} \cap U_{j}$, and

$$
\boldsymbol{g}_{i j}\left(\mathbf{s}^{\prime} \oplus \mathbf{s}^{\prime \prime}\right)=\left(\mathbf{s}^{\prime}+h_{j}\left(\mathbf{s}^{\prime \prime}\right)-h_{i}\left(\mathbf{s}^{\prime \prime}\right)\right) \oplus \mathbf{s}^{\prime \prime} .
$$

But, by definition of the operation of Hom $\left(E^{\prime \prime}, E^{\prime}\right)$ on $E^{\prime \prime} \oplus E^{\prime}$, this shows that the fibre space $E$ is represented by the 1-cocycle $\left\{\boldsymbol{h}_{j}-\boldsymbol{h}_{i}\right\}$, and so corresponds to $\delta\left(I^{\prime \prime}\right)$.

Proposition 3. Let $0 \rightarrow E^{\prime} \rightarrow E \rightarrow E^{\prime \prime} \rightarrow 0$ be an extension of $E^{\prime \prime}$ by $E^{\prime}$, and let $0 \leftarrow E^{\prime *} \leftarrow E^{*} \leftarrow E^{\prime \prime *} \leftarrow 0$ be the dual extension of $E^{\prime *}$ by $E^{\prime \prime *}$. Let

$$
\xi \in H^{1}\left(X, \operatorname{Hom}\left(E^{\prime \prime}, E^{\prime}\right)\right) \text { and } \eta \in H^{1}\left(X, \operatorname{Hom}\left(E^{\prime *}, E^{\prime \prime *}\right)\right)
$$

be the corresponding elements given by Proposition 2. Then $\eta=-\xi^{*}$, where $\xi^{*}$ is the element which corresponds to $\xi$ under the canonical isomorphism: Hom ( $E^{\prime \prime}$, $\left.E^{\prime}\right) \cong \operatorname{Hom}\left(E^{\prime *}, E^{\prime \prime}\right)$.

Proof. We use the same notation as before, so that $E$ is defined by the coordinate transformations

$$
\boldsymbol{g}_{i j}=u_{i}^{-1} u_{j}: E_{i j}^{\prime} \oplus E_{i j}^{\prime \prime} \rightarrow E_{i j}^{\prime} \oplus E_{i j}^{\prime \prime} .
$$

Then $E^{*}$ will be defined by

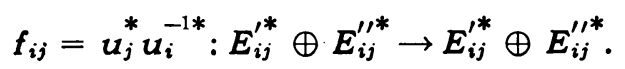


Hence, if $\left\{\boldsymbol{h}_{j}-\boldsymbol{h}_{\boldsymbol{i}}\right\}$ is a representative 1 -cocycle for $\xi,\left\{\boldsymbol{h}_{\boldsymbol{i}}^{*}-\boldsymbol{h}_{j}^{*}\right\}$ is a representative 1 -cocycle for $\eta$. Hence $\eta=-\xi^{*}$.

In future we shall identify the sheaves Hom $\left(E^{\prime \prime}, E^{\prime}\right)$ and Hom $\left(E^{\prime *}, E^{\prime \prime *}\right)$ under the canonical isomorphism, and the result of Proposition 3 can then be written as $\eta=-\xi$.

Finally we introduce the following notation. Let $Y$ be a complex analytic sub-manifold of $X$, and let $E$ be a vector bundle over $X$. We denote by $E_{Y}$ the restriction of $E$ to $Y$, and by $E_{Y}$ the sheaf of germs of cross-sections of $E_{Y}$. Let $O_{Y}$ be the sheaf of germs of holomorphic functions on $Y$, then $E_{Y}$ is a sheaf of $O_{Y}$-modules and we have

$$
E_{Y} \cong E \otimes{ }_{o} O_{Y}
$$

In the usual way we regard $E_{Y}$ as a sheaf of $O$-modules on $X$, zero outside $Y$. Note that $E_{Y}$ is not the sheaf on $Y$ induced from $E$ by the injection $Y \rightarrow X$, but only a quotient of this sheaf.

2. Complex analytic connections. As before let $X$ be a complex manifold, and let $G$ be a connected complex Lie group. Let $P$ be a principal (complex analytic) bundle over $X$ with group $G$. Then $P$ is itself a complex manifold, locally isomorphic with $X \times G$, and $G$ operates on $P$ as a group of fibre-preserving complex analytic homeomorphisms: $P \times G \rightarrow P$. Unless otherwise stated a group will always mean a connected complex Lie group, and a bundle will always mean a complex analytic bundle.

Let $T=T(X)$ be the (contravariant) tangent vector bundle of $X$, and let $B$ be the tangent vector bundle of $P$. Since $G$ operates on $P$ it also operates on $B: B \times G \rightarrow B$. We put $Q=B / G$, so that a point of $Q$ is a field of tangent vectors to $P$, defined along one of its fibres, and invariant under $G$. We shall show that $Q$ has a natural vector bundle structure over $X$.

Let $x_{0} \in X$, and let $U$ be a sufficiently small open neighborhood of $x_{0}$ so that there exists a cross-section of $P$ over $U, s: U \rightarrow P$. Let $B_{s}$ be the restriction of $B$ to $s(U)$, then there is a natural (1-1) correspondence between $Q_{U}$ and $\mathfrak{B}_{s}, \phi_{s}: Q_{U} \rightarrow \bigotimes_{s} ; \phi_{s}$ assigns to each invariant vector field along $P_{x}$ its value at $s(x)$. Since $s: U \rightarrow s(U)$ is a complex analytic homeomorphism, the vector bundle structure of $B_{s}$ defines a vector bundle structure of $Q_{U}$. It remains to show that this structure is independent of the choice of $s$. But, if $s$ and $s^{\prime}$ are two cross-sections of $P$ over $U$, there exists a unique complex analytic map $g: U \rightarrow G$ such that

$$
s(x) \cdot g(x)=s^{\prime}(x) \quad \text { for } x \in U .
$$

Then $g$ induces an isomorphism of vector bundles $g: B_{s} \rightarrow B_{s^{\prime}}$, and we have the commutative diagram

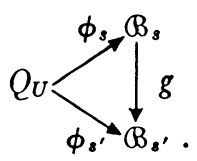


Thus the vector bundle structure of $Q$ over $X$ is well-defined.

Let $\mathcal{F}$ denote the sub-bundle of $B$ formed by vectors tangential to the fibres of $P$. Then $G$ operates on $\mathcal{F}$, and we put $R=\mathscr{F} / G$. If $\phi_{s}$ is the map defined above, then restricting to $R_{U}$, we get

$$
\phi_{s}: R_{U} \rightarrow \mathcal{F}_{s} .
$$

Since $\mathcal{F}_{s}$ is a sub-bundle of $\mathbb{B}_{s}$ it follows at once that $R$ is a sub-bundle of $Q$.

The projection $\pi: P \rightarrow X$ induces a map $\pi: Q \rightarrow T$. From the definition of the vector bundle structure of $Q$ it follows that $\pi: Q \rightarrow T$ is a homomorphism of vector bundles. Moreover, if $T_{s}$ denotes the tangent bundle of $s(U)$, then $\mathfrak{B}_{s}=\mathcal{F}_{s} \oplus T_{s}$, and we have the commutative diagram:

$$
\begin{aligned}
0 \rightarrow R_{U} & \rightarrow Q_{U} \rightarrow T_{U} \rightarrow 0 \\
\downarrow & \quad \downarrow \quad \downarrow \\
0 \rightarrow F_{s} & \rightarrow Q_{s} \rightarrow T_{s} \rightarrow 0 .
\end{aligned}
$$

From this we deduce that $0 \rightarrow R \rightarrow Q \rightarrow T \rightarrow 0$ is an exact sequence of vector bundles over $X$.

We now examine the bundle $R$ more closely. Let $L=L(G)$ be the Lie algebra of $G$. As a vector space, $L$ is the tangent space to $G$ at the identity. By left (right) translation $L$ is then isomorphic with the vector space $V_{l}\left(V_{r}\right)$ of left (right) invariant vector fields on $G$. $G$ operates by right (left) translations on $V_{l}\left(V_{r}\right)$, and by the adjoint representation on $L$. If $l \in L, \lambda \in V_{l}$, $\rho \in V_{r}$ are corresponding elements, then $\operatorname{ad}(g) l, \lambda g^{-1}, g \rho$ are corresponding elements $(g \in G)$. In the principal bundle $P$ each fibre $P_{x}$ can be identified with $G$ up to a left translation. Hence a tangent vector at the point $p$, tangential to the fibre, defines a unique left-invariant vector field on $G$. This establishes the isomorphism $\mathscr{F} \cong P \times L$. The operation of $G$ on $\mathfrak{F}$ induces an operation on $P \times L$, which is given by:

$$
(p \times l) \cdot g=\left(p g \times \operatorname{ad}\left(g^{-1}\right) l\right) .
$$

Hence $R=\mathcal{F} / G \cong P \times{ }_{G} L$, where $P \times{ }_{G} L$ is the identification space defined by (1). By definition however $P \times{ }_{G} L$ is the vector bundle with fibre $L$ associated to $P$ by the adjoint representation of $G$. We shall denote this vector bundle by $L(P)$. Although we shall not require it $L(P)$ is in fact a bundle of Lie algebras, each fibre $L(P)_{x}=L\left(P_{x}\right)$ being a Lie algebra isomorphic with $L(G)$.

We summarize our results as follows:

TheOREM 1. Let $P$ be a principal bundle over $X$ with group $G$. Then there exists a canonical exact sequence $Q(P)$ of vector bundles over $X$ :

$$
0 \rightarrow L(P) \rightarrow Q \rightarrow T \rightarrow 0,
$$

where $T$ is the tangent bundle of $X, L(P)$ is the bundle associated to $P$ by the adjoint representation of $G$, and $Q$ is the bundle of invariant tangent vector fields on $P$. 
Definition. A connection in the principal bundle $P$ is a splitting of the exact sequence $Q(P)$.

By Proposition 2 the extension $a(P)$ defines an element

$$
a(P) \in H^{1}(X, \operatorname{Hom}(T, L(P)),
$$

and $Q(P)$ is a trivial extension if and only if $a(P)=0$. If we denote by $\boldsymbol{\Omega}^{p}$ the sheaf of germs of holomorphic differential forms of degree $p$ on $X$, then $\boldsymbol{\Omega}^{1}=T^{*}$ and Hom $(T, L(P)) \cong L(P) \otimes \Omega^{1}$. Hence, identifying these sheaves under this isomorphism, we can state our result in the following form:

Theorem 2. A principal bundle $P$ over $X$ defines an element

$$
a(P) \in H^{1}\left(X, L(P) \otimes \mathbf{\Omega}^{1}\right) .
$$

$P$ has a connection if and only if $a(P)=0$.

Remark. As we observed in $\S 1$, every extension is trivial in the differentiable case, or in the complex case if $X$ is a Stein manifold. Hence in both these cases a connection always exists.

The sequence $Q(P)$ has certain functorial properties which we now give.

(1) Let $P$ be a principal bundle over $X$ with group $G$, and let $h: G \rightarrow G^{\prime}$ be a homomorphism. Then $h$ induces a principal bundle $P^{\prime}$ with group $G^{\prime}$, and $h: P \rightarrow P^{\prime}$ induces a map of the tangent bundles $h: B \rightarrow B^{\prime}$. Moreover $h$ commutes with the action of $G$ in the sense that $h(b \cdot g)=h(b) \cdot h(g)$. Hence we get a map $h: Q \rightarrow Q^{\prime}$, and it is easy to show that this is compatible with the vector bundle structures, i.e. that it is a homomorphism. Also, restricted to $L(P), h$ induces the natural homomorphism $h: L(P) \rightarrow L^{\prime}\left(P^{\prime}\right)$. Hence we get a homomorphism of exact sequences:

$$
\alpha(h): Q(P) \rightarrow a\left(P^{\prime}\right),
$$

in which of course $T$ is mapped by the identity onto $T$.

(2) Let $f: X^{\prime} \rightarrow X$ be a complex analytic map, and let $P$ be a principal bundle over $X$ with group $G$. Then $f$ induces over $X^{\prime}$ a principal bundle $P^{\prime}$ with group $G$, such that we have a commutative diagram

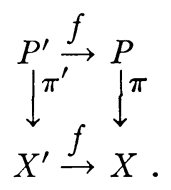

$f: P^{\prime} \rightarrow P$ induces a map $f: \mathbb{B}^{\prime} \rightarrow B$ which commutes with the operation of $G$, and so we get a homomorphism $\left({ }^{3}\right) f: Q \rightarrow Q^{\prime}$. This gives a homomorphism of exact sequences:

${ }^{(3)}$ This is a homomorphism between vector bundles on two different spaces $X^{\prime}, X\left(f: X^{\prime} \rightarrow X\right.$ being given), but the definition of such a homomorphism presents no difficulty. 


$$
Q(f): Q\left(X^{\prime}, P^{\prime}\right) \rightarrow Q(X, P),
$$

in which $T^{\prime} \rightarrow T$ is the homomorphism of tangent bundles induced by $f: X^{\prime} \rightarrow X$.

The functorial properties of $Q(P)$ give rise to corresponding properties of $a(P)$.

(1) Let the notation be as in (1) above, and let

$$
h^{\sharp}: H^{1}\left(X, L(P) \otimes \Omega^{1}\right) \rightarrow H^{1}\left(X, L^{\prime}\left(P^{\prime}\right) \otimes \Omega^{1}\right)
$$

be the homomorphism induced by the sheaf homomorphism $h: L(P) \rightarrow L^{\prime}\left(P^{\prime}\right)$. Then $a\left(P^{\prime}\right)=h^{\sharp} a(P)$.

(2) Let the notation be as in (2) above, and let

$$
f^{\#}: H^{1}\left(X, L(P) \otimes \boldsymbol{\Omega}^{1}(X)\right) \rightarrow H^{1}\left(X^{\prime}, L\left(P^{\prime}\right) \otimes \mathbf{\Omega}^{1}\left(X^{\prime}\right)\right)
$$

be the homomorphism induced by the sheaf homomorphism

$$
f: L(P) \otimes \Omega^{1}(X) \rightarrow L\left(P^{\prime}\right) \otimes \Omega^{1}\left(X^{\prime}\right) .
$$

Then $a\left(P^{\prime}\right)=f^{\sharp} a(P)$.

We omit the proof of these two statements since we shall later give an explicit form for $a(P)$, in terms of the coordinate transformations of $P$, and (1) and (2) will be immediate consequences.

A case of special interest arises in (2) when $X^{\prime}=Y$ is a submanifold of $X$, and $f: Y \rightarrow X$ is the inclusion map. Then $P^{\prime}=P_{Y}$ is the restriction of $P$ to $Y$, and $\mathfrak{a}\left(P_{Y}\right)$ is the part of $a(P)_{Y}$ lying over $T(Y)$ (which is a sub-bundle of $\left.T(X)_{Y}\right)$.

Theorem 2 becomes particularly simple if $G$ is abelian. In this case the adjoint representation is trivial, so that $L(P) \cong O^{k}$, where $k=\operatorname{dim} G$. Hence $a(P) \in H^{1}\left(X, O^{k} \otimes \boldsymbol{\Omega}^{1}\right)$. Suppose in particular that $X$ is a compact Kähler manifold; then $a(P)=\left(a_{1}, \cdots, a_{k}\right)$, where $a_{i} \in H^{1}\left(X, \mathbf{\Omega}^{1}\right)$ defines a cohomology class of type $(1,1)$. When $G$ is a complex torus, Theorem 2 reduces to a result of Blanchard [3]. The case where $G=C^{*}$ will be discussed later.

3 . The characteristic ring. In this section we relate the treatment of $\$ 2$ with the ordinary theory of differentiable connections, as expounded for instance in Chern [6].

First we show how to calculate the obstruction $a(P)$ explicitly in terms of the coordinate transformations of $P$. Let $P$ be defined with respect to the covering $\left\{U_{i}\right\}$ by the coordinate transformations $\left\{g_{i j}\right\}$, i.e. we have the local isomorphisms $u_{i}: U_{i} \times G \rightarrow P \mid U_{i}$, and

$$
u_{i}^{-1} u_{j}: U_{i} \cap U_{j} \times G \rightarrow U_{i} \cap U_{j} \times G
$$

is given by $u_{i}^{-1} u_{j}(x \times g)=\left(x \times g_{i j}(x) g\right)$. The map $u_{i}$ induces an isomorphism of tangent bundles, and since it commutes with the operation of $G$ we obtain the isomorphism

$$
\mathfrak{u}_{i}: T_{i} \oplus L_{i} \rightarrow Q_{i}
$$


where $L$ denotes the trivial bundle $X \times L$ and $L_{i}, T_{i}, Q_{i}$ denote the restrictions of $L, T, Q$ to $U_{i}$. Thus, over $U_{i}$, we can lift the identity endomorphism of $T$ to the element $a_{i}: T_{i} \rightarrow Q_{i}$, by defining $a_{i}(t)=\hat{u}_{i}(t \oplus 0)$. Put $a_{i j}=a_{j}-a_{i}$ : $T_{i j} \rightarrow Q_{i j}$. Then $\left\{a_{i j}\right\}$ is a representative 1-cocycle for $a(P)$.

Let $\rho: T(G) \rightarrow G \times L, \lambda: T(G) \rightarrow G \times L$ be the isomorphisms obtained by right and left translation respectively. Then $\rho, \lambda$ are both elements of $H^{0}(G$, Hom $(T(G), L))$, where $L$ denotes the sheaf corresponding to the trivial bundle $G \times L$. The maps $g_{i j}: U_{i} \cap U_{j} \rightarrow G$ then induce elements $\rho_{i j}, \lambda_{i j}$ $\in \Gamma\left(U_{i} \cap U_{j}\right.$, Hom $\left.(T, L)\right)$, where $T=T(X)$ and $L$ now denotes the sheaf corresponding to the trivial bundle $X \times L$. Then

$$
\begin{aligned}
{\hat{u_{i}}}^{-1} a_{i j}(t) & =\hat{u}_{i}^{-1} \hat{u}_{j}(t \oplus 0)-(t \oplus 0) \\
& =\left(t \oplus \rho_{i j}(t)\right)-(t \oplus 0) \\
& =\left(0 \oplus \rho_{i j}(t)\right) .
\end{aligned}
$$

If we denote by the same symbol $\hat{u}_{i}$ the induced isomorphism of $L_{i}$ onto $L(P)_{i}$, this equation can be written

$$
a_{i j}=\hat{u}_{i} \cdot \rho_{i j}
$$

Since $\rho_{i j}, \lambda_{i j}$ are related by the equation $\rho_{i j}=\operatorname{ad}\left(g_{i j}\right) \lambda_{i j}$, (2) may be replaced by

$$
a_{i j}=\hat{u}_{j} \cdot \lambda_{i j} \text {. }
$$

If $a(P)=0$ then we have the coboundary equation

$$
a_{i j}=f_{j}-f_{i}
$$

where $f_{i} \in \Gamma\left(U_{i}\right.$, Hom $\left.(T, L(P))\right)$. If we put $\theta_{i}=\hat{u}_{i}^{-1} f_{i}$, then

$$
\theta_{i} \in \Gamma\left(U_{i}, \operatorname{Hom}(T, L)\right)
$$

and the coboundary equation becomes

$$
\rho_{i j}=\operatorname{ad}\left(g_{i j}\right) \theta_{j}-\theta_{i}
$$

or

$$
\lambda_{i j}=\theta_{j}-\operatorname{ad}\left(g_{j i}\right) \theta_{i} .
$$

These equations are the ones usually taken to define a connection (cf. [6]), but the reader should watch carefully for differences of notation.

We recall next that, if $E$ is a complex analytic vector bundle, a generalized Dolbeault isomorphism can be defined for the sheaf of differential forms with coefficients in $E$. For the details see for instance [13]. If $A=\Sigma A^{p, q}$ is the bigraded sheaf of germs of $C^{\infty}$ differential forms on $X$, we put $A(E)=E \otimes_{0} A$. The operator $d^{\prime \prime}$ is a coboundary operator of the complex $H^{0}\left(X, E \otimes_{0} A\right)$, and we denote the component of type $(p, q)$ of the cohomology of this complex by $H^{p, q}(X, E)$. Then the Dolbeault isomorphism takes the form 


$$
H^{p, q}(X, E) \cong H^{q}\left(X, E \otimes \mathbf{\Omega}^{p}\right) .
$$

Applying this in particular to the vector bundle $L(P)$, we obtain the isomorphisms

$$
H^{p, q}(X, L(P)) \cong H^{q}\left(X, L(P) \otimes \Omega^{p}\right) .
$$

Suppose now that we are given a $C^{\infty}$ connection in $P$, i.e. a $C^{\infty}$ set of $\theta_{i}$ satisfying (4) or (5). Then the curvature $\Theta$ of this connection is given (cf. [6]) by

$$
\Theta=d \theta_{i}+\frac{1}{2}\left[\theta_{i}, \theta_{i}\right] \quad \text { in } U_{i} .
$$

If $\theta_{i}$ is of type $(1,0)$ then $(7)$ shows that $\Theta^{1,1}$, the component of type $(1,1)$ of $\Theta$ is given by

$$
\Theta^{1,1}=d^{\prime \prime} \theta_{i}
$$

From (4) and (8) we see that the $d^{\prime \prime}$-cohomology class $\left[\Theta^{1,1}\right]$ of $\Theta^{1,1}$ is independent of the choice of connection (provided this is of type $(1,0)$ ). In fact equation (4) asserts that $\left[\Theta^{1,1}\right]$ corresponds, in the Dolbeault isomorphism (6), to the element $a(P) \in H^{1}\left(X, L(P) \otimes \boldsymbol{\Omega}^{1}\right)$. Thus we have

Proposition 4. Let $\theta$ be a $C^{\infty}$ connection of type $(1,0)$ in $P$, and let $\Theta^{1,1}$ denote the component of type $(1,1)$ of the corresponding curvature, $\left[\Theta^{1,1}\right]$ $\in H^{1,1}(X, L(P))$ its $d^{\prime \prime}$-cohomology class. Then $\left[\Theta^{1,1}\right]$ corresponds to $a(P)$ $\in H^{1}\left(X, L(P) \otimes \boldsymbol{\Omega}^{1}\right)$ under the Dolbeault isomorphism.

From now on, in this section, we shall assume that the group $G$ is either semi-simple or is $G L_{r}$. Let $G_{1}$ be a maximal compact subgroup of $G$, then $G_{1}$ is a real Lie group. We have the following relations between $G$ and $G_{1}$.

(i) Every $C^{\infty}$ principal bundle with group $G$ is equivalent to a bundle with group $G_{1}$.

(ii) $L(G)$ is the complexification of $L\left(G_{1}\right)$.

(iii) Let $F_{1}: L\left(G_{1}\right) \otimes_{R} \cdots \otimes_{R} L\left(G_{1}\right) \rightarrow R$ be a symmetric $R$-homomorphism invariant under ad $\left(G_{1}\right)$-briefly $F_{1}$ is called an invariant polynomial of $G$ (cf. [6]); then $F_{1}$ induces a $C$-homomorphism $F: L(G) \otimes_{C} \cdots \otimes_{C} L(G) \rightarrow C$, and the relation $F_{1} \leftrightarrow F$ sets up a (1-1) correspondence between the invariant polynomials of $G_{1}$ and those invariant polynomials of $G$ whose restriction to $L\left(G_{1}\right)$ is real.

If $P_{1}$ is a $C^{\infty}$ principal bundle with group $G_{1}$, it induces a $C^{\infty}$ principal bundle $P$ with group $G$. A $C^{\infty}$ connection $\theta_{1}$ in $P_{1}$ induces a $C^{\infty}$ connection $\theta$ in $P$. Then we have the following result, due to Singer [14] and Nakano [11]:

Proposition 5. Let $P$ be a complex analytic principal bundle with group $G, P_{1}$ a $C^{\infty}$ principal bundle with group $G_{1}$ which induces $P$. Then there is a 
unique $C^{\infty}$ connection $\theta_{1}$ in $P_{1}$, such that the induced $C^{\infty}$ connection $\theta$ in $P$ is of type $(1,0)$. Moreover the curvature of $\theta$ is of type $(1,1)$.

Let $\theta_{1}$ be a connection in $P_{1}$, as in Proposition 5. Let $\Theta_{1}, \Theta$ be the corresponding curvatures in $P_{1}, P$, and let $F_{1}, F$ be invariant polynomials of $G_{1}, G$ which correspond as in (iii) above. Then, if $[\omega]$ denotes the cohomology class defined by a closed differential form $\omega$,

$$
\begin{aligned}
{\left[F_{1}\left(\Theta_{1}\right)\right] } & =\left[F_{1}\left(\Theta_{1}, \Theta_{1}, \cdots, \Theta_{1}\right)\right] \in H^{*}(X, R), \\
{[F(\Theta)] } & =[F(\Theta, \Theta, \cdots, \Theta)] \in H^{*}(X, C),
\end{aligned}
$$

and

$$
[F(\Theta)]=i^{*}\left[F_{1}\left(\Theta_{1}\right)\right], \quad \text { where } i^{*}: H^{*}(X, R) \rightarrow H^{*}(X, C)
$$

is induced by the natural embedding $i: \boldsymbol{R} \rightarrow \boldsymbol{C}$. By a basic theorem (cf. [6]) the characteristic cohomology ring of $P$ (with real coefficients), denoted by $R(P)$, consists of the elements $\left[F_{1}\left(\Theta_{1}\right)\right]$, for all invariant polynomials $F_{1}$ of $G_{1}$. We denote by $\mathfrak{C}(P)$ the characteristic cohomology ring of $P$ with complex coefficients, an element $\alpha \in \mathfrak{e}(P)$ being a linear combination $\alpha=\sum_{j} C_{j} i *\left(\beta_{j}\right)$, with $\beta_{j} \in R(P)$, the coefficients $C_{j}$ being complex numbers. It then follows from the preceding statements that $\mathfrak{C}(P)$ consists precisely of the elements $[F(\Theta)]$, for all invariant polynomials $F$ of $G$.

If $F$ is any invariant polynomial of $G$ it defines a sheaf homomorphism $L(P) \otimes L(P) \otimes \cdots \otimes L(P) \rightarrow O$, and so we can define (by cup-product multiplication)

$$
F(a(P))=F(a(P), a(P), \cdots a(P)) \in H^{k}\left(X, \mathbf{\Omega}^{k}\right),
$$

where $k$ is the degree of $F$. Hence, from Propositions 4 and 5, and using the fact that the Dolbeault isomorphism preserves multiplicative structures up to sign (cf. [7]), we deduce:

Theorem 3. Let $X$ be a compact Kähler manifold, $P$ a complex analytic principal bundle over $X$ with group $G$ (semi-simple or $\left.G L_{r}\right)$. Let

$$
a(P) \in H^{1}\left(X, L(P) \otimes \boldsymbol{\Omega}^{1}\right)
$$

be the element defined in Theorem 2. Then, under the operation of the invariant polynomials of $G, a(P)$ generates the characteristic cohomology ring of $P$ (with complex coefficients).

The restriction on $X$ to be compact Kähler in the theorem is needed so that we can identify $H^{k}\left(X, \mathbf{\Omega}^{k}\right)$ with a subspace of $H^{2 k}(X, \mathrm{C})$.

Combining Theorems 2 and 3 we obtain:

Theorem 4. Let $X$ be a compact Kähler manifold, $P$ a complex analytic principal bundle over $X$ with group $G$ (semi-simple or $\left.G L_{r}\right)$. If $P$ has a complex 
analytic connection, then the characteristic cohomology ring of $P$ (with complex coefficients) is zero.

The problem of reversing Theorem 4, and obtaining criteria for the existence of a complex analytic connection in terms of the characteristic ring, will be examined in $\$ \S 7$ and 8 . We may state at once however that, unless further restrictions are imposed, the converse of Theorem 4 is false.

4. The $D$-functor. If $P$ is a principal bundle with group $G L_{r}$, then there is a (1-1) correspondence between $P$ and $E$, the associated vector bundle. From $E$ we can construct the sheaf $E$ and conversely. Hence a vector bundle has two natural generalizations: (i) to a bundle with different structure group, (ii) to an arbitrary coherent sheaf. So far we have concentrated on (i); we now turn to (ii), and we show how to generalize the obstruction $a(P)$ in this direction.

Let $X$ be a complex manifold, $O$ the sheaf of germs of holomorphic functions on $X$. Let $S$ be a coherent sheaf of $O$-modules on $X$ (cf. [12]). Then we define a new sheaf $D(\boldsymbol{S})$ as follows:

(i) as a sheaf of $C$-modules $D(\boldsymbol{S})=\boldsymbol{S} \oplus \boldsymbol{S} \otimes \mathbf{\Omega}^{1}$,

(ii) if $\boldsymbol{\alpha}=\mathbf{s} \oplus \boldsymbol{\beta} \in D(\boldsymbol{S})_{x}, \boldsymbol{f} \in \boldsymbol{O}_{x}$, we define $\boldsymbol{f} \cdot \boldsymbol{\alpha}=\mathbf{f} \boldsymbol{s} \oplus(\boldsymbol{f} \hat{\beta}+\mathbf{s} \otimes \boldsymbol{d} \boldsymbol{f})$. We have to verify that $D(\boldsymbol{S})_{x}$ is now an $\boldsymbol{O}_{x}$-module. Clearly $\boldsymbol{f} \cdot \boldsymbol{\alpha}$ is bilinear, and we have

$$
f(g \alpha)=f g s \oplus(f g \xi+g s \otimes d f+f s \otimes d g)=(f g) \alpha .
$$

Since the operations of $\boldsymbol{O}_{x}$ on $D(\boldsymbol{S})_{x}$ are clearly continuous, it follows that $D(\boldsymbol{S})$ is a sheaf of $\boldsymbol{O}$-modules. Moreover we have an exact sequence

$$
\mathrm{B}(\mathrm{S}): 0 \rightarrow S \stackrel{\boldsymbol{i}}{\rightarrow} D(\boldsymbol{S}) \stackrel{\boldsymbol{p}}{\rightarrow} \boldsymbol{S} \rightarrow 0,
$$

where $i: \boldsymbol{\beta} \rightarrow \boldsymbol{O} \oplus \boldsymbol{\beta}, \boldsymbol{p}: \mathbf{s} \oplus \boldsymbol{\beta} \rightarrow \mathbf{s}$ are both $\boldsymbol{O}$-homomorphisms. Since $\boldsymbol{S}$ is coherent, $S \otimes \Omega^{1}$ is coherent, and hence $D(S)$ is coherent (cf. [12]).

Proposition 6. $B$ is a functor.

Proof. If $\phi: S \rightarrow S^{\prime}$ is an $\boldsymbol{O}$-homomorphism we must define a homomorphism of exact sequences $B(\boldsymbol{\phi}): B(\boldsymbol{S}) \rightarrow B\left(\boldsymbol{S}^{\prime}\right)$. Let $\boldsymbol{\phi} \otimes 1: \mathbf{S} \otimes \boldsymbol{\Omega}^{1} \rightarrow \boldsymbol{S}^{\prime} \otimes \boldsymbol{\Omega}^{1}$ be the homomorphism induced by $\boldsymbol{\phi}$, and define $D(\boldsymbol{\phi}): D(\boldsymbol{S}) \rightarrow D\left(\boldsymbol{S}^{\prime}\right)$ by

$$
D(\phi) \cdot s \oplus \underline{\beta}=\phi s \oplus(\phi \otimes 1 \cdot \hat{\beta}) \text {. }
$$

Then $D(\phi)$ is an $\boldsymbol{O}$-homomorphism, since

$$
\begin{aligned}
D(\phi) \cdot f(s \oplus \beta) & =\phi f s \oplus \phi \otimes 1 \cdot(f \beta+s \otimes d f) \\
& =f \phi s \oplus(f(\phi \otimes 1) \cdot \beta+\phi s \otimes d f) \\
& =f \cdot D(\phi) \cdot(s \oplus \beta) .
\end{aligned}
$$

Then we have a homomorphism of exact sequences $B(\phi)$ : 


$$
\begin{aligned}
& 0 \longrightarrow S \otimes \Omega^{1} \longrightarrow D(S) \longrightarrow S \longrightarrow 0 \\
& \downarrow \phi \otimes 1 \quad \downarrow D(\phi) \quad \downarrow \phi \\
& 0 \longrightarrow S^{\prime} \otimes \boldsymbol{\Omega}^{1} \longrightarrow D\left(\boldsymbol{S}^{\prime}\right) \longrightarrow \boldsymbol{S}^{\prime} \longrightarrow 0 \text {. }
\end{aligned}
$$

Moreover $B(\phi)$ clearly has the necessary functorial properties.

Proposition 7. $B$ is an exact functor.

Proof. Let $0 \rightarrow S^{\prime} \rightarrow S \rightarrow S^{\prime \prime} \rightarrow 0$ be an exact sequence. We have to show that $0 \rightarrow B\left(\boldsymbol{S}^{\prime}\right) \rightarrow B(\boldsymbol{S}) \rightarrow \circledast\left(\boldsymbol{S}^{\prime \prime}\right) \rightarrow 0$ is also exact. But, as a sheaf of $C$-modules, $D(\boldsymbol{S})=\boldsymbol{S} \oplus \boldsymbol{S} \otimes \boldsymbol{\Omega}^{1}$. Hence, we have a $C$-module isomorphism,

$$
\Theta(S) \cong \Theta\left(S^{\prime}\right) \oplus \otimes\left(S^{\prime \prime}\right),
$$

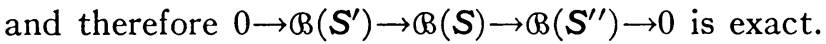

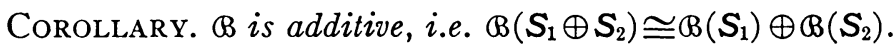

Proposition 8. If $\boldsymbol{S}$ is locally free, so is $D(\mathbf{S})$.

Proof. Since $\boldsymbol{S}$ is locally free, $\boldsymbol{S} \otimes \boldsymbol{\Omega}^{1}$ is also locally free. Since $\boldsymbol{S}$ is locally free the extension $B(S)$ is locally trivial (cf. $\S 1$ ), and so we have the local $\boldsymbol{O}$-isomorphism $D(\boldsymbol{S}) \cong \boldsymbol{S} \oplus \boldsymbol{S} \otimes \boldsymbol{\Omega}^{1}$. Hence $D(\boldsymbol{S})$ is locally free.

Thus, if $E$ is a vector bundle over $X$, there exists a vector bundle which we may denote by $D(E)$, and an exact sequence of vector bundles.

$$
\Theta(E): 0 \rightarrow E \otimes T^{*} \rightarrow D(E) \rightarrow E \rightarrow 0 .
$$

The extension $B(E)$ defines an element $b(E) \in H^{1}\left(X\right.$, Hom $\left.\left(E, E \otimes T^{*}\right)\right)$. Since Hom $\left(E, E \otimes T^{*}\right) \cong E n d E \otimes \Omega^{1}$, we may regard $b(E)$ as an element of $H^{1}\left(X\right.$, End $\left.\boldsymbol{E} \otimes \boldsymbol{\Omega}^{1}\right)$. We shall show that $b(E)$ is essentially the same as the element $a(P)$ defined by the corresponding principal bundle.

Proposition 9. Let $E$ be a vector bundle over $X, P$ the corresponding principal bundle. Then End $E \cong L(P)$.

Proof. If $V$ is a vector space, and $G=\operatorname{Aut}(V)$ is the group of all automorphisms of $V$, then we have a canonical isomorphism $L(G)=$ End $(V)$. Now let $E$ be a vector bundle, $P$ the corresponding principal bundle with group $G=G L_{r}$. Then $G$ operates on itself by inner automorphisms, and so we obtain a bundle $G(P)$ associated to $P$. This is a bundle of groups, and for each $x, G(P)_{x}$ operates in a canonical manner on $P_{x}$ by left translation. Hence $G(P)_{x} \cong$ Aut $E_{x}$, and so $L\left(G(P)_{x}\right) \cong$ End $E_{x}$. But $L\left(G(P)_{x}\right) \cong L(P)_{x}$, both being isomorphic to the vector space of right invariant vector fields on $P_{x}$. Hence $L(P)_{x} \cong$ End $E_{x}$. Moreover the isomorphism is canonical and preserves the vector bundle structure. Hence $L(P) \cong$ End $E$.

From Proposition 9 we deduce the isomorphism

$$
H^{1}\left(X, L(P) \otimes \Omega^{1}\right) \cong H^{1}\left(X, \text { End } E \otimes \Omega^{1}\right),
$$


so that $a(P)$ and $b(E)$ can be regarded as elements of the same group.

THEOREM 5. Let $E$ be a vector bundle over $X, P$ the associated principal bundle. Let $a(P), b(E)$ be the obstruction elements defined by the extensions $a(P)$, $B(E)$ respectively. Then $a(P)=-b(E)$.

Proof. Let $P$ be defined, with respect to the covering $\left\{U_{i}\right\}$, by the coordinate transformations $\left\{g_{i j}\right\}$, with local isomorphisms

$$
u_{i}: U_{i} \times G \rightarrow P \mid U_{i}, \quad u_{1}^{-1} u_{j}(x \times g)=\left(x \times g_{i j}(x) g\right) .
$$

Let $V$ be the vector space on which $G$ operates, so that $E=P \times{ }_{G} V$, and let $V$ denote the sheaf corresponding to the trivial bundle $X \times V$. We shall use the same symbol $u_{i}$ to denote the induced sheaf homomorphisms:

$$
\begin{aligned}
& u_{i}: V_{i} \rightarrow E_{i}, \\
& u_{i}: V_{i} \otimes \Omega_{i}^{1} \rightarrow E_{i} \otimes \Omega_{i}^{1} .
\end{aligned}
$$

Then we can define a $C$-homomorphism of sheaves over $U_{i}$,

$$
d_{i}: E_{i} \rightarrow E_{i} \otimes \Omega_{i}^{1},
$$

by $\boldsymbol{d}_{i} \mathbf{s}=\boldsymbol{u}_{i} d u_{i}^{-1} \mathbf{s}$. We now define $\boldsymbol{\psi}_{i}: E_{i} \rightarrow D(E)_{i}$, by $\boldsymbol{\psi}_{i} \mathbf{s}=\mathbf{s} \oplus \boldsymbol{d}_{i} \mathbf{s}$. The definition of the $O$-module structure of $D(E)$ was precisely chosen so that $\Psi_{i}$ should be an $\boldsymbol{O}$-homomorphism. This shows that $\left\{\boldsymbol{b}_{i j}\right\}$ is a representative cocycle for $b(E)$, where $b_{i j} \in \Gamma\left(U_{i} \cap U_{j}\right.$, Hom $\left.\left(E, E \otimes \boldsymbol{\Omega}^{1}\right)\right)$ is given by

$$
b_{i j} s=d_{j} s-d_{i} s \text {. }
$$

Hence

$$
\begin{aligned}
& u_{i}^{-1} b_{i j} u_{i} \cdot v=g_{i j} d\left(g_{j i} \cdot v\right)-d v \\
&=g_{i j} d\left(\hat{g_{i j}}\right) \cdot v \\
&=-\left(d g_{i j}\right)-1 \\
& g_{i j}^{-1} \cdot v .
\end{aligned}
$$

But $\left(\boldsymbol{d} \boldsymbol{g}_{i j}\right) \boldsymbol{g}_{i j}^{-1}=\boldsymbol{g}_{i j}$, in the notation of $\S 3$. Here we are identifying the three sheaves Hom $\left(\boldsymbol{V}, \boldsymbol{V} \otimes \boldsymbol{\Omega}^{1}\right)$, Hom $(\boldsymbol{T}, \boldsymbol{L})$, and $\boldsymbol{L} \otimes \boldsymbol{\Omega}^{1}$ under the canonical isomorphisms. If we also identify $\operatorname{Hom}\left(E, E \otimes \Omega^{1}\right), \operatorname{Hom}(T, L(P)), L(P) \otimes \Omega^{1}$ under the canonical isomorphisms, equations (2) and (9) show that $b_{i j}=-a_{i j}$. Hence $b(E)=-a(P)$ as required.

More generally, it is possible to relate the functors $Q(P)$ and $B(E): \leftrightarrow(E)$ is canonically isomorphic with a subsequence of $Q(P)^{*} \otimes E$. We shall not require this result but we wish to point it out because, in conjunction with Proposition 2, it helps to explain the minus sign in Theorem 5.

The behavior of $B(E)$ under tensor products is given by the following result:

Proposition 10. $B\left(E \otimes E^{\prime}\right)=B(E) \otimes E^{\prime}+E \otimes B\left(E^{\prime}\right)$, where each side repre- 
sents an extension of $E \otimes E^{\prime} \otimes T^{*}$ by $E \otimes E^{\prime}$, and + denotes the sum of two extensions.

This proposition is not difficult to prove by direct verification. As we do not need it however we omit the proof. A corollary of Proposition 10, which is also immediate from the explicit calculation of $b(E)$ in terms of the coordinate transformations, is the following:

Proposition 11. $b\left(E \otimes E^{\prime}\right)=b(E) \otimes I^{\prime}+I \otimes b\left(E^{\prime}\right)$, both sides being in $H^{1}\left(X\right.$, End $\left.\left(E \otimes E^{\prime}\right) \otimes \Omega^{1}\right)$, and $I, I^{\prime}$ denoting the identity endomorphisms of $E, E^{\prime}$.

5. Chern classes. In $\S 3$ we showed how the obstruction $a(P)$ of a principal bundle $P$ generated the characteristic cohomology ring of $P$ (under suitable restrictions). We shall now give an alternative treatment in the case of the group $G L_{r}(C)$. This is based on the treatment of Chern classes given by Hirzebruch [9]. We suppose throughout that $X$ is a compact Kähler manifold.

Proposition 12. Let $E$ be a line-bundle over $X$, then $b(E) \in H^{1}\left(X, \boldsymbol{\Omega}^{1}\right)$ is given by $b(E)=-2 \pi i c(E)$, where $c(E)$ is the Chern class of $E$.

Proof. Let $E$ be defined by the coordinate transformations $\left\{g_{i j}\right\}$. Then we have shown that $b(E)$ is represented by the cocycle $-d g_{i j} \cdot g_{i j}^{-1}=-d \log g_{i j}$; since $E$ is a line bundle, End $E$ is a trivial line-bundle, and so, in the notation of $\S 4$, we do not have to distinguish between $L$ and $L(P)$. Now we have the commutative diagram of sheaves on $X$ :

$$
\begin{aligned}
0 \rightarrow Z & \rightarrow O \stackrel{e^{2 \pi i}}{\longrightarrow} O^{*} \rightarrow 0 \\
& \downarrow \\
0 \rightarrow C \rightarrow O & \downarrow
\end{aligned}
$$

where $\boldsymbol{Z}, \boldsymbol{C}$ denote the constant sheaves of integers and complex numbers respectively, and $O^{*}$ denotes the multiplicative sheaf of germs of nonzero holomorphic functions; the vertical homomorphism $O^{*} \rightarrow \boldsymbol{\Omega}^{1}$ is $(1 / 2 \pi i) d \log$. From this we obtain the cohomology diagram:

$$
\begin{aligned}
& H^{1}\left(X, O^{*}\right) \stackrel{\alpha}{\rightarrow} H^{2}(X, Z) \\
& \downarrow \delta \quad \downarrow \beta \\
& H^{1}\left(X, \Omega^{1}\right) \stackrel{\gamma}{\rightarrow} H^{2}(X, C) .
\end{aligned}
$$

In this $\gamma$ is an injection (since $X$ is compact Kähler), and we identify $H^{1}\left(X, \Omega^{1}\right)$ with its image in $H^{2}(X, C)$. The line-bundle $E$ defines an element $\epsilon \in H^{1}\left(X, O^{*}\right)$, and by definition $\alpha(\epsilon)=z(E)$, where $z(E)$ denotes the integral Chern class of $E$. Thus $\beta \alpha(\epsilon)=c(E)$ is the Chern class of $E$ with complex coefficients. On the other hand $(1 / 2 \pi i)\left\{d \log g_{i j}\right\}$ is a representative cocycle for $\delta(\epsilon)$. Hence $b(E)=-2 \pi i \delta(\epsilon)=-2 \pi i c(E)$, by the commutativity of the square. 
Let $\Sigma_{k}$ be the polynomial of degree $k$ which assigns to any $g \in G L_{r}$ the $k$ th elementary symmetric function $\sigma_{k}$ of the characteristic roots of $g$, and let $S_{k}$ be the corresponding invariant polynomial of $G L_{r}$ obtained by polarization: thus $S_{k}(g, g, \cdots, g)=\Sigma_{k}(g)$. If $E$ is a split vector bundle, i.e. such that its structure group can be reduced to the triangular group $\Delta_{r}$ (with zeros below the diagonal), then the diagonal terms of the coordinate transformations define $r$ line-bundles $E_{1}, \cdots, E_{r}$, and we have

$$
c_{k}(E)=\sigma_{k}\left[c\left(E_{1}\right), \cdots, c\left(E_{r}\right)\right],
$$

where $c_{k}(E)$ is the $k$ th Chern class of $E$ with complex coefficients (cf. [9]). On the other hand, as in $\S 3$, we may form $\dot{S}_{k}[b(E)] \in H^{k}\left(X, \boldsymbol{\Omega}^{k}\right)$, which we regard as a cohomology class in $H^{2 k}(X, C)$. Since $g_{i j}$ may now be taken triangular, $d g_{i j} \cdot g_{i j}^{-1}$ will also be triangular, and hence we obtain the formula

$$
S_{k}[b(E)]=\sigma_{k}\left[c\left(E_{1}\right), \cdots, c\left(E_{r}\right)\right] .
$$

Combining this with Proposition 12 we obtain:

Proposition 13. Let $E$ be a split vector bundle, then $S_{k}[b(E)]=(-2 \pi i)^{k} c_{k}(E)$ gives the kth Chern class of $E$ in terms of $b(E)$.

If $E$ is a general vector bundle over $X$ we can consider the associated bundle $Y$ with fibre the flag manifold $G L_{r} / \Delta_{r}$. Then $Y$ is also a compact Kähler manifold (cf. [4]). If $p: Y \rightarrow X$ is the bundle projection we can consider the induced bundle $\hat{E}=p^{-1}(E)$ over $Y$; $\hat{E}$ is then split (cf. [9]). Now, by the functorial properties of $b(E)$ (which we stated for $a(P)$, and so hold for $b(E)$ by Theorem 5 ), we have

$$
b(\hat{E})=p^{*} b(E) .
$$

On the other hand we have $c_{k}(\hat{E})=p^{*} c_{k}(E)$, and finally $p^{*}: H^{q}(X, C)$ $\rightarrow H^{q}(Y, C)$ is injective (cf. [9]). From all this, together with Proposition 13 we obtain $\left({ }^{4}\right)$ :

THEOREM 6. Let E be a vector bundle on a compact Kähler manifold. Then the kth Chern class of $E$ is given by

$$
c_{k}(E)=(-2 \pi i)^{-k} S_{k}[b(E)] .
$$

6. Line-bundles. Let $V$ be a complex vector space, $\Sigma$ the corresponding projective space, $\pi: V-\{0\} \rightarrow \Sigma$ the canonical projection. $C^{*}$ operates by right multiplication on $V-\{0\}$ :

$$
\lambda: v \rightarrow v \lambda, \quad v \in V-0, \quad \lambda \in C^{*} .
$$

Hence $P=V-\{0\}$ is a principal bundle over $\Sigma$ with group $C^{*}$. Let $E$ be the

(4) Using a result of Borel-Hirzebruch we can deduce Theorem 3 from Theorem 6 py considering all complex analytic representations of $G$ in $G L_{r}(C)$. 
associated line-bundle, then $E=[-H]$ or $E^{*}=[H]$, where $[H]$ denotes the line-bundle corresponding to a hyperplane (cf. [9]). We propose to investigate the functor $Q(P)$ in this case.

Let $B$ be the tangent bundle of $P$; by translating the tangent vector $b$ at $p$ to $b_{0}$ at 0 , and then identifying the tangent space to $V$ at 0 with $V$ itself, we obtain an isomorphism $B \cong P \times V$. $C^{*}$ operates on $B$, and the corresponding operation on $P \times V$ is given by:

$$
(p \times v) \lambda=(p \lambda \times v \lambda), \quad \quad p \in P, v \in V, \lambda \in C^{*} .
$$

Hence $Q=B / C^{*} \cong P \times V / C^{*} \cong P \times{ }_{c^{*}} V$, where $P \times{ }_{C^{*}} V$ is the bundle with fibre $V$ associated to $P$ by the representation $\rho$ :

$$
\rho(\lambda) v=\lambda^{-1} v, \quad v \in V, \lambda \in C^{*} .
$$

Thus $Q \cong E^{*} \otimes V$, where $V$ denotes the trivial bundle $\Sigma \times V$, and the isomorphism is a vector bundle isomorphism over $\Sigma$.

Since $C^{*}$ is abelian $L(P)$ is a trivial line bundle, denoted by 1 . Hence, using the isomorphism $Q \cong E^{*} \otimes V, a(P)$ gives the exact sequence:

$$
0 \rightarrow 1 \rightarrow E^{*} \otimes V \rightarrow T \rightarrow 0 .
$$

Take the tensor product of (10) with $E$, and we get the exact sequence:

$$
0 \rightarrow E \rightarrow V \rightarrow T \otimes E \rightarrow 0 .
$$

Since $1=L(P)$ was the sub-bundle of $Q$ consisting of invariant vector fields tangential to the fibres of $P$, it follows that the homomorphism $E \rightarrow V$ of (11) is precisely the natural embedding of $E$ in $\Sigma \times V$ :

$$
(p, c) \rightarrow \pi(p) \times p c, \quad(p, c) \in P \times c^{*} C=E .
$$

Suppose now that $X$ is a nonsingular algebraic variety of dimension $r$ embedded in the projective space $\Sigma$ of dimension $n$. Let $G(r+1, n+1)$ denote the Grassmannian of $(r+1)$-dimensional vector sub-spaces of $V$, or equivalently the Grassmannian of $r$-dimensional projective sub-spaces of $\Sigma$. Let $\Sigma_{x}$ be the tangent $r$-dimensional projective space of $X$ at $x$. Then $x \rightarrow \Sigma_{x}$ defines a mapping $X \rightarrow G(r+1, n+1)$, which induces an $(r+1)$-dimensional vector bundle $W$ over $X$. We call $W$ the tangent bundle of the embedding of $X$ in $\Sigma$. From the definition it follows that $W$ is a sub-bundle of the trivial bundle $X \times V$. Now from $\S 2, a\left(P_{X}\right)$ is that part of the sequence $a(P)_{X}$ which lies above $T(X)$. Hence from (10) we see that $Q\left(P_{X}\right)$ is isomorphic to the exact sequence:

$$
0 \rightarrow 1_{X} \rightarrow E_{X}^{*} \otimes W \rightarrow T(X) \rightarrow 0 .
$$

With a slight change of notation we state this as:

THEOREM 7. Let $X$ be a nonsingular algebraic variety embedded in projective space, $E$ the line-bundle over $X$ corresponding to minus a hyperplane section, $W$ 
the tangent bundle of the embedding and $T$ the tangent bundle of $X$. Further let $P$ be the principal bundle corresponding to $E$; then we have the exact sequence of vector bundles over $X\left(^{5}\right)$ :

$$
a(P) \otimes E: 0 \rightarrow E \rightarrow W \rightarrow T \otimes E \rightarrow 0 .
$$

The tangent bundle $W$ of the embedding of $X$ in projective space was that originally used by Todd [15] to define the characteristic classes of $X$, and his formula is contained in the following corollary of Theorem 7 (cf. Nakano $[10])$.

Corollary. Let $X, E, W, T$ be as in Theorem 7, then the Chern classes $c_{k}(T)$ of $X$ are given by

$$
c_{k}(T)=c_{k}\left(W \otimes E^{*}\right) .
$$

Proof. From the differentiable point of view every extension is trivial; hence, regarded as differentiable bundles, we have the isomorphism $W \otimes E^{*}$ $\cong T \oplus 1$. Applying the Whitney sum formula for Chern classes (cf. [9]) the required result follows immediately.

In particular we may remark that (10) gives the formula for the Chern classes of a projective space at once!

We observe finally that the extensions (10), (11), (12) are never trivial (if $\operatorname{dim} X>0$ ). The extension (10) corresponds to $a(P) \in H^{1}\left(\Sigma, \mathbf{\Omega}^{1}\right)$, and we have shown that $a(P)=-b(E)=2 \pi i c(E) \neq 0$. The same holds if we replace $\Sigma$ by $X$, showing that (12) is nontrivial $\left({ }^{(}\right)$. This confirms the uniqueness of direct decomposition for vector bundles in this special case (cf. [1] for the general theorem).

7. Representations of the fundamental group. We assume from now on that $X$ is connected. Let $\rho: \pi_{1}(X) \rightarrow G$ be a representation of the fundamental group of $X$ into the connected complex Lie group $G$. The universal covering space $\widetilde{X}$ of $X$ is a principal bundle over $X$ with group $\pi_{1}(X)$, and the representation $\rho$ induces therefore a principal bundle $P$ over $X$ with group $G$. We have $P=X \times_{\pi_{1}} G$, where $\pi_{1}$ operates on the left on $G$ :

$$
\alpha \cdot g=\rho(\alpha) g, \quad \alpha \in \pi_{1}, g \in G .
$$

There are various alternative ways of looking at those principal bundles which arise from a representation of the fundamental group, and we now discuss these.

Let $P$ be a principal bundle over $X$ with group $G$. Then $P$, the sheaf of germs of cross-sections of $P$, is a sheaf of sets, and $G$ operates on $P: P \times G \rightarrow P$. Hence we may form the quotient sheaf $P / G$. If $U$ is an open set on $X$, and $s \in \Gamma(U, P)$, we have a local isomorphism $U \times G \rightarrow P \mid U$ given by

(5) This exact sequence is due to Serre (unpublished) and Nakano [10].

(6) Cf. Nakano [10]. 


$$
x \times g \rightarrow s(x) g .
$$

Hence $s$ defines a splitting of the exact sequence $a\left(P_{U}\right)$. Moreover $s g$, where $g \in G$, defines the same splitting. Hence a global section of $P / G$ defines a spliting of $a(P)$.

Definition. An integrable connection in the principal bundle $P$ is one which arises from a global section of $P / G$.

Just as in the real differentiable case, we can introduce the curvature $\Theta$ of a complex analytic connection, and $\Theta=0$ will be the necessary and sufficient condition for the connection to be integrable (cf. [6]). In particular, since $\Theta$ is a holomorphic differential form of degree two with coefficients in $L(P), \Theta$ vanishes identically if $X$ is of complex dimension one. Hence on an algebraic curve (without singularities) every connection is integrable.

If $G$ is a complex Lie group we denote by $G^{*}$ the corresponding abstract group with the discrete topology.

Definition. $P^{*}$ is a discrete form of the principal bundle $P$ over $X$ if

(i) $P^{f}$ is a topological principal bundle over $X$ with the discrete group $G^{\prime}$ as structure group,

(ii) there is a continuous bijective bundle map $J: P^{\sharp} \rightarrow P$.

Condition (i) means that, locally, $P^{\#}$ has the topology of $X \times G^{*}$. Condition (ii) asserts that $P^{\sharp}$ is obtained from $P$ by imposing a new topology.

Proposition 14. Let $P$ be a principal bundle over $X$ with group $G$. Then the following properties are equivalent to one another:

(a) $P$ arises from a representation of the fundamental group,

(b) $P$ can be expressed in terms of constant coordinate transformations,

(c) $P$ has an integrable connection,

(d) $P$ has a discrete form $P^{\#}$.

Proof. We shall prove the implications:

$$
(\text { a) } \rightarrow(\text { b) } \rightarrow(\text { c) } \rightarrow(\text { d) } \rightarrow \text { (a). }
$$

Since $\pi_{1}(X)$ is a discrete group, $\tilde{X}$ the universal covering of $X$, has constant coordinate transformations. Hence the same is true for a bundle induced by a representation. Next, if $P$ satisfies (b), the locally constant cross-sections of $P$ are well-defined, and give a global section of $P / G$, so that $P$ has an integrable connection. Suppose now that $P$ satisfies (c), so that we are given a section $s \in \Gamma(X, P / G)$. Let $\sigma: P \rightarrow P / G$ be the canonical projection, and let $P^{\sharp} \subset P$ be the subspace defined by $P^{\sharp}=\sigma^{-1} s(X) . P$ is a topological space, oper . ated on by $G$, with a projection $P \rightarrow X$, and a continuous map $P \rightarrow P$ (assigning to each germ $\boldsymbol{p}_{\boldsymbol{x}}$ of $P$ its value at $x$ ). Hence $P^{\boldsymbol{t}}$ has a topology, a projection $P \rightarrow X$, and a continuous map $J: P \rightarrow P$; moreover it is closed under the operation of $G$. $P^{\star}$ is a discrete form of $P$, and so $P$ satisfies (d). Finally let $P^{t}$ be a discrete form of $P$. Since $G^{*}$ is discrete, each connected component of $P^{f}$ is a covering space of $X$, and so there exists a homomorphism $\rho^{*}: \pi_{1}(X) \rightarrow G^{t}$ 
inducing $P^{\sharp}$. Let $\rho: \pi_{1}(X) \rightarrow G$ be the homomorphism corresponding to $\rho^{\sharp}$, then $P$ arises from the representation $\rho$. This completes the proof of the proposition.

Applying Proposition 14, Theorem 2, and the fact that we have no integrability condition for a curve, we obtain:

Theorem 8. Let $P$ be a principal bundle over an algebraic curve $X$. Then $P$ arises from a representation of the fundamental group if and only if $a(P)=0$, where $a(P) \in H^{1}\left(X, L(P) \otimes \Omega^{1}\right)$ is the invariant defined in Theorem 2.

Let $L^{*}(P)$ be the vector bundle dual to $L(P)$, and let $X$ be an algebraic curve. Then, by Serre's duality theorem [13], $H^{1}\left(X, L(P) \otimes \boldsymbol{\Omega}^{1}\right)$ is dual to $H^{0}\left(X, L^{*}(P)\right)$, i.e. to $\Gamma L^{*}(P)$. Thus $a(P)$ may be regarded as an element of $\left(\Gamma L^{*}(P)\right)^{*}$, the vector space dual to $\Gamma L^{*}(P)$. If $G$ is semi-simple, or is $G L_{r}$, then $L^{*}(G)$ is canonically isomorphic to $L(G)$ (by the Killing form), and so $L^{*}(P)$ is canonically isomorphic with $L(P)$. In particular, in the case of $G L_{r}, L(P)$, and so $L^{*}(P)$, is canonically isomorphic with End $E$, where $E$ is the vector bundle associated to $P$ (Proposition 9). Hence, using Theorem 5, we can restate Theorem 8 in this case as follows:

TheOREM 9. Let $E$ be a vector bundle over an algebraic curve $X$. Then $E$ defines an element $b(E) \in(\Gamma \text { End } E)^{*}$, and $E$ arises from a representation of the fundamental group if and only if $b(E)=0$.

Theorem 9 suggests that we should study the endomorphisms of a vector bundle, and here we have the following result:

Proposition 15. Let $E$ be an indecomposable vector bundle over a connected compact complex manifold. Then every endomorphism of $E$ is either nilpotent or is an automorphism.

This is a special case of a more general result for coherent sheaves, for the proof of which we refer to [1]; we recall only that $E$ is indecomposable if it has no nontrivial direct decomposition: $E=E_{1} \oplus E_{2}$. Proposition 15 can be given a slightly different form as follows. Let $A$ be a finite dimensional algebra over $C$ with the following properties:

(i) $A$ has a unit element $I$,

(ii) the nilpotent elements in $A$ form a subalgebra $N$,

(iii) as a vector space $A=\{I\} \oplus N$, where $\{I\}$ is the subspace of dimension one generated by $I$.

For brevity we shall say that $A$ is a special algebra. Then we have:

Proposition 16. Let $E$ be a vector bundle over a connected compact complex manifold $X$. Then $E$ is indecomposable if and only if $\Gamma$ End $E$ is a special algebra.

Proof. Let $E$ be indecomposable, and let $\phi \in \Gamma$ End $E$. For each $x \in X$ $\phi_{x} \in$ End $E_{x}$, and the characteristic equation of $\phi_{x}$ has coefficients which are 
holomorphic functions of $x$. Since $X$ is compact and connected these coefficients are constant, and so the characteristic roots are constants. If $\phi$ had two distinct characteristic roots $\lambda, \mu$, then $\phi-\lambda I \in \Gamma$ End $E$ would be neither an automorphism nor nilpotent, contradicting Proposition 15. Hence all the characteristic roots of $\phi$ are equal, and so the nilpotent endomorphisms of $E$ are precisely those of trace zero. This proves that $\Gamma$ End $E$ is a special algebra. Conversely, let $\Gamma$ End $E$ be a special algebra $A$, and suppose if possible that $E=E_{1} \oplus E_{2}$ is a nontrivial direct decomposition of $E$. Let $I_{1}, I_{2}$ be the identity endomorphisms of $E_{1}, E_{2}$; then $\lambda_{1} I_{1} \oplus \lambda_{2} I_{2}$ is nilpotent if and only if $\lambda_{1}=\lambda_{2}=0$. Thus $I_{1}, I_{2}$ generate a subspace of $A(\bmod N)$ of dimension two, contradicting condition (iii) for a special algebra. Hence $E$ must be indecomposable.

If $E$ is any vector bundle over a compact complex manifold, and if $E=E_{1} \oplus E_{2} \oplus \cdots \oplus E_{q}$ is a Remak decomposition of $E$, i.e. a decomposition in which all the $E_{i}$ are indecomposable and nonzero, then the factors $E_{i}$ are unique to within isomorphism ([1, Theorem 3]). Also, since $B(E)$ and so $b(E)$ is additive (Corollary to Proposition 7), we have $b(E)=b\left(E_{1}\right) \oplus b\left(E_{2}\right)$ $\oplus \cdots \oplus b\left(E_{q}\right)$; this is also clear from the coordinate transformations. Hence, from Theorem 9, we obtain:

Proposition 17. Let $E$ be a vector bundle over an algebraic curve, and let $E=E_{1} \oplus E_{2} \oplus \cdots \oplus E_{q}$ be a Remak decomposition of $E$. Then $E$ arises from $a$ representation of the fundamental group if and only if all the $E_{i}$ arise from the fundamental group.

In view of Proposition 17 we may restrict attention to indecomposable vector bundles. For these we have Proposition 16, and we shall now evaluate the scalar product $\langle b(E), \phi\rangle$ in the two cases (i) when $\phi$ is the identity, (ii) when $\phi$ is nilpotent.

Proposition 18. Let $E$ be a vector bundle over a curve $X$. Let $I$ be the identity endomorphism of $E$, and $\phi$ a nilpotent endomorphism of $E$. Then

(i) $\langle b(E), I\rangle=-2 \pi i \operatorname{deg} E$,

(ii) $\langle b(E), \phi\rangle=0$,

where deg $E$ denotes the degree of $c_{1}(E)$.

Proof. First we remark that, for any $\psi \in \Gamma$ End $E$,

$$
\langle b(E), \psi\rangle_{\mu}=\operatorname{trace}(\psi b(E)),
$$

where $\mu \in H^{1}\left(X, \boldsymbol{\Omega}^{1}\right)$ is the canonical generator, and $\psi(b) E \in H^{1}\left(X\right.$, End $\left.E \otimes \boldsymbol{\Omega}^{1}\right)$ is obtained by endomorphism multiplication. Hence, since $\operatorname{deg} E \cdot \mu=c_{1}(E)$, (i) follows immediately from Theorem 6 . It remains to prove (ii). Consider the sequence $E, \phi(E), \phi^{2}(E), \cdots$. Since $\phi$ is nilpotent this sequence terminates, i.e. $\phi^{n}(E)=0$ for some $n$. In general $\phi^{k}(E)$ will not be a sub-bundle of $E$, since $\phi^{k}\left(E_{x}\right)$ need not be of constant dimension. However, since $X$ is a curve, $\phi^{k}(E)$ generates a sub-bundle $E_{k}$ of $E$ such that $\phi^{k}\left(E_{x}\right)=\left(E_{k}\right)_{x}$ for all except 
a finite number of points $x$ (cf. [2]). Thus we have a sequence of sub-bundles of $E$ :

$$
E=E_{0} \supset E_{1} \supset E_{2} \cdots \supset E_{n-1} \supset E_{n}=0
$$

with the property that $\phi\left(E_{k}\right) \subset E_{k+1}$, or equivalently the induced homomorphism $\phi_{k}: E_{k} / E_{k+1} \rightarrow E_{k} / E_{k+1}$ is zero. Since $B$ is an exact functor, or alternatively from the coordinate transformations, we see that $b(E) \in H^{1}(X$, End $\left.E \otimes \boldsymbol{\Omega}^{1}\right)$ is in the image of $H^{1}\left(X, E_{n d}{ }^{\prime} \otimes \boldsymbol{\Omega}^{1}\right)$, where End ${ }^{\prime} E$ is the subsheaf of End $E$ which preserves the sequence (13). Hence

$$
\text { trace }(\phi b(E))=\Sigma_{k} \text { trace }\left(\phi_{k} b\left(E_{k} / E_{k+1}\right)\right)=0,
$$

since $\phi_{k}=0$. Thus $\langle b(E), \phi\rangle=0$ as required.

Combining Theorem 9 with Propositions 16 and 18 we obtain:

Proposition 19. Let $E$ be an indecomposable vector bundle over an algebraic curve. Then $E$ arises from a representation of the fundamental group if and only if $\operatorname{deg} E=0$.

Finally, from Propositions 17 and 19 we obtain Weil's Theorem (cf. [17]).

THEOREM 10. Let $E$ be a vector bundle over an algebraic curve, and let $E=E_{1} \oplus E_{2} \oplus \cdots \oplus E_{q}$ be a Remak decomposition of $E$. Then $E$ arises from $a$ representation of the fundamental group if and only if $\operatorname{deg} E_{i}=0, i=1,2, \cdots, q$.

8. Higher dimensional varieties. In this section we shall discuss problems concerning connections, when the base space is of dimension greater than one.

Let $X$ be a nonsingular algebraic variety embedded in the projective space $\Sigma$. We denote by $X_{n}$ the intersection of $X$ with a general hypersurface of degree $n$ in $\Sigma$. If $E$ is a vector bundle on $X$, we write $E_{n}$ for $E_{X_{n}}$, the restriction of $E$ to $X_{n}$. Further we denote by $J$ the line-bundle on $X$ corresponding to a hyperplane section, so that $\left(^{7}\right) J=\left[X_{1}\right], J^{n}=\left[X_{n}\right]$. If $S$ is any coherent sheaf of $O$-modules on $X$ we use the notation of [12] and put

$$
S(n)=S \otimes o J^{n} \text {. }
$$

If $E$ is any vector bundle on $X$, then we have an exact sequence of sheaves (cf. [12]),

$$
0 \rightarrow E(-n) \rightarrow E \rightarrow E_{n} \rightarrow 0 .
$$

Finally we recall that, if $n$ is sufficiently large, $H^{q}(X, E(n))=0$ for $q \geqq 1$, and $H^{q}(X, E(-n))=0$ for $q<\operatorname{dim} X$. The first of these two is proved in [5], and the second follows from the first by Serre's duality theorem [13]. Using (14), and the vanishing of these cohomology groups, one can reduce the dimension of the base space. We use this method for the next two propositions.

( 7 ) As usual $[D]$ denotes the line-bundle corresponding to the divisor $D$. 
Proposition 20. Let $X$ be an algebraic variety of dimension greater than one, and let $E$ be an indecomposable vector bundle over $X$. Then, for sufficiently large $n, E_{n}$ is indecomposable.

Proof. Apply (14) to the vector bundle End $E$. Since (End $\left.E_{n}\right) \cong E n d E_{n}$, we get the exact sequence of sheaves

$$
0 \rightarrow(\text { End } E)(-n) \rightarrow \text { End } E \rightarrow \text { End } E_{n} \rightarrow 0 .
$$

Since $\operatorname{dim} X \geqq 2$, we have

$$
H^{0}(X,(\text { End } E)(-n))=H^{1}(X,(\text { End } E)(-n))=0,
$$

for sufficiently large $n$. Hence, for this $n$, the cohomology sequence gives:

$$
H^{0}(X, \text { End } E) \cong H^{0}\left(X_{n}, \text { End } E_{n}\right),
$$

i.e.

\section{$\Gamma$ End $E \cong \Gamma$ End $E_{n}$.}

Applying Proposition 16 the required result follows at once.

Proposition 21. Let $X$ be an algebraic variety of dimension greater than two, and let $P$ be a principal bundle over $X$. Then $P$ has a connection if and only if $P_{n}$, the restriction of $P$ to $X_{n}$, has a connection for sufficiently large $n$.

Proof. Let $T=T(X)$ denote the tangent bundle of $X$. Apply (14) to the vector bundle $L(P) \otimes T^{*}$ where, as in $\S 2, L(P)$ is the vector bundle associated to $P$ by the adjoint representation. Since $\operatorname{dim} X \geqq 3$, we have, for large $n$,

$$
H^{q}\left(X, L(P) \otimes T^{*}(-n)\right)=0 \quad \text { for } q=1,2 .
$$

Hence, from the cohomology sequence of (14), we obtain

$$
H^{1}\left(X, L(P) \otimes T^{*}\right) \cong H^{1}\left(X_{n}, L(P)_{n} \otimes T_{n}^{*}\right)
$$

If $T\left(X_{n}\right)$ denotes the tangent bundle of $X_{n}$, we have the exact sequence of vector bundles on $X_{n}$ :

$$
0 \rightarrow T\left(X_{n}\right) \rightarrow T(X)_{n} \rightarrow J_{n}^{n} \rightarrow 0,
$$

and the dual exact sequence:

$$
0 \rightarrow J_{n}^{-n} \rightarrow T^{*}(X)_{n} \rightarrow T^{*}\left(X_{n}\right) \rightarrow 0 .
$$

This is essentially what is known as the "adjunction formula" (cf. [16]); $J_{n}^{n}=\left[X_{n}\right]_{n}$ is the characteristic line-bundle of $X_{n}$ in $X$. Operate on (17) by $L(P)_{n} \otimes$, and take the corresponding exact sequence of sheaves:

$$
0 \rightarrow L(P)(-n)_{n} \rightarrow L(P)_{n} \otimes T^{*}(X)_{n} \rightarrow L(P)_{n} \otimes T^{*}\left(X_{n}\right) \rightarrow 0 .
$$


Now apply (14) to the vector bundle $L(P) \otimes J^{-n}$, and we get the exact sequence of sheaves:

$$
0 \rightarrow L(P)(-2 n) \rightarrow L(P)(-n) \rightarrow L(P)(-n)_{n} \rightarrow 0 .
$$

Since $\operatorname{dim} X \geqq 3$, we have

$$
H^{2}(X, L(P)(-2 n))=H^{1}(X, L(P)(-n))=0,
$$

for sufficiently large $n$. Hence, for this $n$, from the cohomology sequence of (19):

$$
H^{1}\left(X, L(P)(-n)_{n}\right)=0 .
$$

From the cohomology sequence of (18) this shows that, for sufficiently large $n$,

$$
H^{1}\left(X_{n}, L(P)_{n} \otimes T^{*}(X)_{n}\right) \rightarrow H^{1}\left(X_{n}, L(P)_{n} \otimes T^{*}\left(X_{n}\right)\right)
$$

is injective. Together with (15) this shows that the homomorphism

$$
i_{n}^{*}: H^{1}\left(X, L(P) \otimes T^{*}(X)\right) \rightarrow H^{1}\left(X_{n}, L(P)_{n} \otimes T^{*}\left(X_{n}\right)\right),
$$

induced by the inclusion $i_{n}: X_{n} \rightarrow X$, is injective for sufficiently large $n$. But, from the functorial properties of $a(P)$ given in $\$ 2$, we have $a\left(P_{n}\right)=i_{n}^{*} \cdot a(P)$. Hence, if $n$ is sufficiently large, $a(P)=0$ if and only if $a\left(P_{n}\right)=0$. Hence, from Theorem 2, $P$ has a connection if and only if $P_{n}$ has a connection.

We shall now construct an example which shows that Proposition 21 does not hold if $\operatorname{dim} X=2$. Our example will also show that the obvious generalization of Weil's Theorem (Theorem 10) does not hold if $\operatorname{dim} X \geqq 2$.

Let $Y$ be a rational curve, $Z$ an elliptic curve, and let $X=Y \times Z$. We choose base points $y_{0} \in Y, z_{0} \in Z$, and we identify $Y$ with $Y \times z_{0}, Z$ with $y_{0} \times Z$. Consider the exact sequence of sheaves on $X$ :

$$
0 \rightarrow[Z] \rightarrow[2 Z] \rightarrow O_{Z} \rightarrow 0 .
$$

This arises in the same way as (14), except that $X_{n}$ is replaced here by the curve $Z$; the restriction of $[Z]$ to $Z$ is the trivial line-bundle, and so the corresponding sheaf is $O_{z}$. The exact cohomology sequence gives

$$
\rightarrow H^{1}(X,[2 Z]) \rightarrow H^{1}\left(Z, O_{Z}\right) \rightarrow H^{2}(X,[Z]) \rightarrow .
$$

By Serre's duality theorem $H^{2}(X,[\boldsymbol{Z}])$ is dual to $H^{0}(X,[\boldsymbol{K}-\boldsymbol{Z}])$ where $K$ is a canonical divisor on $X$. But $K=-2 Z$, and $H^{0}(X,[-3 Z])=0$. Hence $H^{1}(X,[\mathbf{2 Z}]) \rightarrow H^{1}\left(Z, O_{Z}\right)$ is surjective, so there is an element $\xi \in H^{1}(X,[\mathbf{2 Z}])$ mapping onto a nonzero element $\eta$ in $H^{1}\left(Z, O_{Z}\right)$ (this being of dimension one since $Z$ is elliptic). Now the elements of $H^{1}(X,[2 Z])$ are in one-toone correspondence with the classes of extensions of $[-Z]$ by $[Z]$ (Proposition 2). Hence there is a 2-dimensional vector bundle $E$ over $X$ such that the exact sequence 


$$
0 \rightarrow[Z] \rightarrow E \rightarrow[-Z] \rightarrow 0,
$$

corresponds to the element $\xi$. We give the properties of $E$ in the following proposition:

Proposition 22. Let $E$ be the vector bundle defined above over the algebraic surface $X$, and let $P$ denote the corresponding principal bundle. Then

(i) the Chern classes of $E$ are all zero,

(ii) $E$ is indecomposable,

(iii) $P$ does not have a connection.

Proof. (i) If $g$ is the cohomology class of the divisor $Z$ on $X$, (20) shows that the Chern polynomial (cf. [9]) of $E$ is $(1+g)(1-g)=1-g^{2}=1$. In other words the Chern classes (of positive dimension) of $E$ are all zero. (ii) Restricting (20) to $Z$ we get the exact sequence:

$$
0 \rightarrow 1_{Z} \rightarrow E_{Z} \rightarrow 1_{Z} \rightarrow 0 .
$$

By construction $\eta \in H^{1}\left(Z, O_{Z}\right)$ is nonzero, and hence (21) is a nontrivial extension. Hence $E_{Z}$ is indecomposable (cf. [2]) and so $E$ is indecomposable. (iii) Restricting (20) to $Y$ we get the exact sequence:

$$
0 \rightarrow[D] \rightarrow E_{Y} \rightarrow[-D] \rightarrow 0,
$$

where $D$ is a point divisor on $Y$. Since $Y$ is a rational curve $H^{1}(Y,[2 D])=0$, this being dual $H^{0}(Y,[-4 D])$. Hence the extension (22) is trivial, and $E_{Y} \cong[D] \oplus[-D]$. Since $\operatorname{deg}[D]=1 \neq 0, b([D]) \neq 0$, and so $b\left(E_{Y}\right) \neq 0$. But $b\left(E_{Y}\right)$ is the image of $b(E)$ in the homomorphism

$$
H^{1}\left(X, \text { End } E \otimes T^{*}(X)\right) \rightarrow H^{1}\left(Y, \text { End } E_{Y} \otimes T^{*}(Y)\right) .
$$

Hence $b(E) \neq 0$, and so by Theorems 2 and $5, P$ does not have a connection.

Proposition 22 shows immediately that Theorem 10 does not generalize to varieties of higher dimension. Also, continuing with the example, if $X_{n}$ is the intersection of $X$ with a general hypersurface of degree $n$, Proposition 20 and Proposition 22 (ii) tell us that $E_{n}$ is indecomposable for sufficiently large $n$. By Proposition 22 (i) the first Chern class of $E_{n}$ is zero, i.e. $\operatorname{deg} E_{n}=0$. Hence by Theorem 10, $P_{n}$ has a connection. But by Proposition 22 (iii) $P$ does not have a connection. Thus Proposition 21 is false if $\operatorname{dim} X=2$.

My thanks are due to J.-P. Serre, S. Nakano and I. M. Singer for many valuable discussions.

\section{REFERENCES}

1. M. F. Atiyah, On the Krull-Schmidt theorem with application to sheaves, Bull. Soc. Math. France vol. 84 (1956) pp. 307-317.

2. M. F. Atiyah, Vector bundles over an elliptic curve, Proc. London Math. Soc. vol. 7 (1957).

3. A. Blanchard, Variétes Kählériennes et espaces fibrés, C.R. Acad. Sci. Paris vol. 234 (1952) pp. 284-286. 
4. - Espaces fibrés Kählériens compacts, C.R. Acad. Sci. Paris vol. 238 (1954) pp. 2281-2283.

5. H. Cartan and J.-P. Serre, Seminaire E.N.S., 1953-1954.

6. S. S. Chern, Differential geometry of fiber bundles, Proceedings of the International Congress of Mathematicians, 1950, pp. 397-411.

7. P. Dolbeault, Thèse (to appear in Ann. of Math.).

8. A. Grothendieck, $A$ general theory of fibre spaces with structure sheaf, University of Kansas, Report No. 4, 1955. 1956.

9. F. Hirzebruch, Neue topologische Methoden in der algebraischen Geometrie, Springer,

10. S. Nakano, Tangential vector bundle and Todd canonical systems of an algebraic variety, Mem. Coll. Sci. Kyoto, Series A vol. 29 (1955) pp. 145-149.

11. - On complex analytic vector bundles, J. Math. Soc. Japan vol. 7 (1955) pp. 1-12.

12. J.-P. Serre, Faisceaux algébriques coherents, Ann. of Math. vol. 61 (1955) pp. 197-278.

13. —, Un théorème de dualitê, Comm. Math. Helv. vol. 29 (1955) pp. 9-26.

14. I. M. Singer, (to appear).

15. J. A. Todd, Invariant and convariant systems on an algebraic variety, Proc. London Math. Soc. (2) vol. 46 (1939) pp. 199-230.

16. — The geometrical invariants of algebraic loci, Proc. London Math. Soc. (2) vol. 45 (1938) pp. 410-424.

17. A. Weil, Generalization de fonctions abeliennes, J. Math. Pures Appl. vol. 17 (1938) pp. 47-87.

InSTITUTE for Advanced Study,

Princeton, N. J. 